\title{
Transmitting Hertzian optical nano-antenna with free-electron feed
}

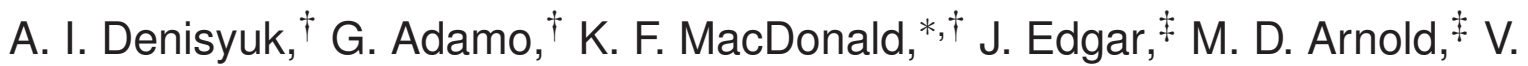 \\ Myroshnychenko, ${ }^{\text {, }}$ M. J. Ford ${ }^{\ddagger}$ F. Javier García de Abajo, ${ }^{, I}$ and N. I. Zheludev ${ }^{\dagger}$ \\ Optoelectronics Research Centre, University of Southampton, Southampton, SO17 1BJ, UK., \\ Department of Physics and Advanced Materials \& Institute for Nanoscale Technology, University \\ of Technology, Sydney, PO Box 123, Broadway, NSW 2007, Australia, and Instituto de Óptica - \\ CSIC, Serrano 121, 28006 Madrid, Spain
}

E-mail: kfm@orc.soton.ac.uk;www.nanophotonics.org.uk

\section{Abstract}

A pair of coupled gold nanorods excited by a beam of free electrons acts as a transmitting Hertzian antenna in the optical part of the spectrum. Significantly enhanced resonant emission is observed from the antenna when the electron beam is injected around the junction between the rods, where the local density of electromagnetic states is elevated.

Structures akin to the dipole antenna introduced by Heinrich Rudolph Hertz around 1886, comprising an arrangement of two metallic rods with a center-fed driven element, have recently attracted considerable attention as potential nanoscale 'optical antennae'. Such antennae would be able to efficiently couple the energy of free-space radiation to a confined region of sub-wavelength size, or to modify the amount and direction of electromagnetic energy emitted into the far-field from nanoscale optical or plasmonic sources. ${ }^{1,2}$ These components are expected to find appli-

\footnotetext{
*To whom correspondence should be addressed

Optoelectronics Research Centre, University of Southampton, Southampton, SO17 1BJ, UK.

†Department of Physics and Advanced Materials \& Institute for Nanoscale Technology, University of Technology, Sydney, PO Box 123, Broadway, NSW 2007, Australia

II Instituto de Óptica - CSIC, Serrano 121, 28006 Madrid, Spain
}

cations in field-enhanced single-molecule spectroscopy ${ }^{3}$ and as elements of future nanophotonic circuits. $^{2,4}$ Thus far, experimental studies of nanoantenna structures have largely been conducted using a combination of optical far-field measurements and near-field scanning probe microscopies to investigate the interplay between plasmonic excitations and light . 5,6 However, It has recently been shown that electron beams, which can of course be focused to nanoscale spots, provide alternative highly localized means of gener-

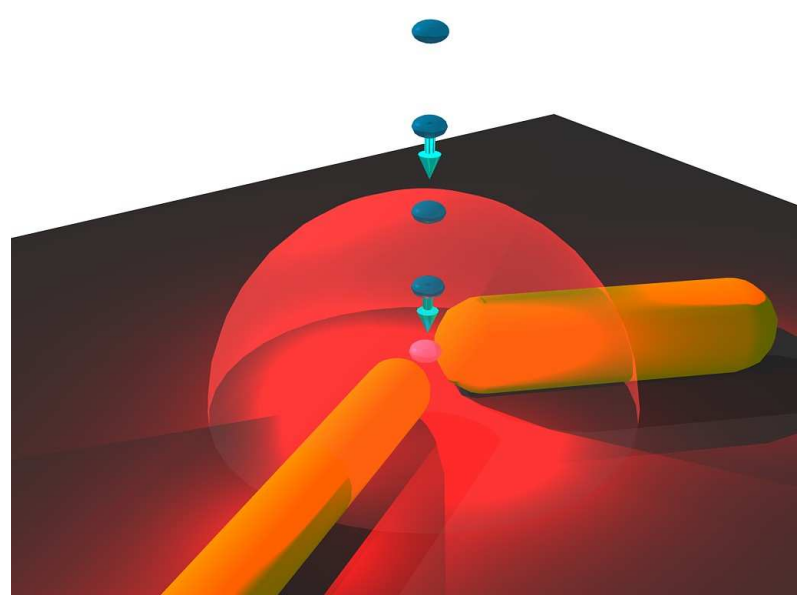

Figure 1: Artist's impression of a transmitting nanoscale optical antenna driven by an electron beam at the junction between rods. A pair of coupled gold nanorods efficiently transfers the energy of electrons focused on the junction into far-field optical radiation. 
ating light and surface plasmons on the nanoscale: the first tunable electron-beam-pumped nanoscale light source has recently been demonstrated, ${ }^{7}$ and spatially-resolved mapping of plasmon modes in metallic nanostructures ${ }^{8-14}$ has been facilitated by the fact that an electron beam impacting on a metal surface acts as a mobile, broadband, nanoscale surface plasmon source. ${ }^{15,16}$ For example, in the case of single gold and silver nanorods such techniques reveal standing wave plasmonic modes resulting from interference between the excited SPPs and back-scattered SPPs reflected from the ends of the rod. ${ }^{10-12}$ Here we report on the experimental realization of transmitting optical antennae based on coupled gold nanorods and pumped by a freeelectron beam. Strongly enhanced antenna efficiency is observed for electron injection points in the vicinity of the junction between rods (Fig. 1).

The antennas were assembled from gold nanorods synthesized using the seed-mediated growth method ${ }^{17,18}$ and then dispersed from colloidal suspension by drop-casting and desiccation on a silicon substrate. Among the randomly distributed nanoparticles, isolated single nanorods and coupled nanorod pairs (of arbitrary mutual orientation) were identified for study. Imaging and parallel analysis of electron-beam-induced radiation emission (EIRE) were performed in a scanning electron microscope (SEM) equipped with a hyperspectral light collection system: ${ }^{8}$ The electron beam was directed onto a samples via a small hole in a parabolic mirror mounted directly above the sample. This collected the light emitted from the sample over approximately half of the available hemispherical solid angle and directed it out of the SEM chamber to a spectrum analyzer with a liquid nitrogen-cooled CCD detector. A hyperspectral image of the sample is built up by scanning it point by point with the focused electron beam and synchronously recording the light emission spectra at every pixel. In the present study, data were recorded for wavelengths between 450 and $850 \mathrm{~nm}$ with an electron acceleration voltage of $40 \mathrm{kV}$ and a beam current of $4.5 \mathrm{nA}$. Separate measurements of substrate (silicon) cathodoluminescent emission enabled background subtraction from the experimental data. This technique relies on the fact that energy is coupled from incident electrons to the plasmonic modes of the antenna structure and subsequently to propagating light modes, which constitute a decay channel for the plasmons. ${ }^{10,19}$ It should be emphasized that EIRE mapping reveals the efficiency with which electron energy is coupled to far-field radiation as a function of electron injection position and not the distribution of light emission, i.e. while the electron-beam excitation is confined to a nanoscale spot, the associated optical emission may come from any part of the structure).

Hyperspectral EIRE mapping of a single, isolated gold nanorod (Fig 2a) reveals the kind
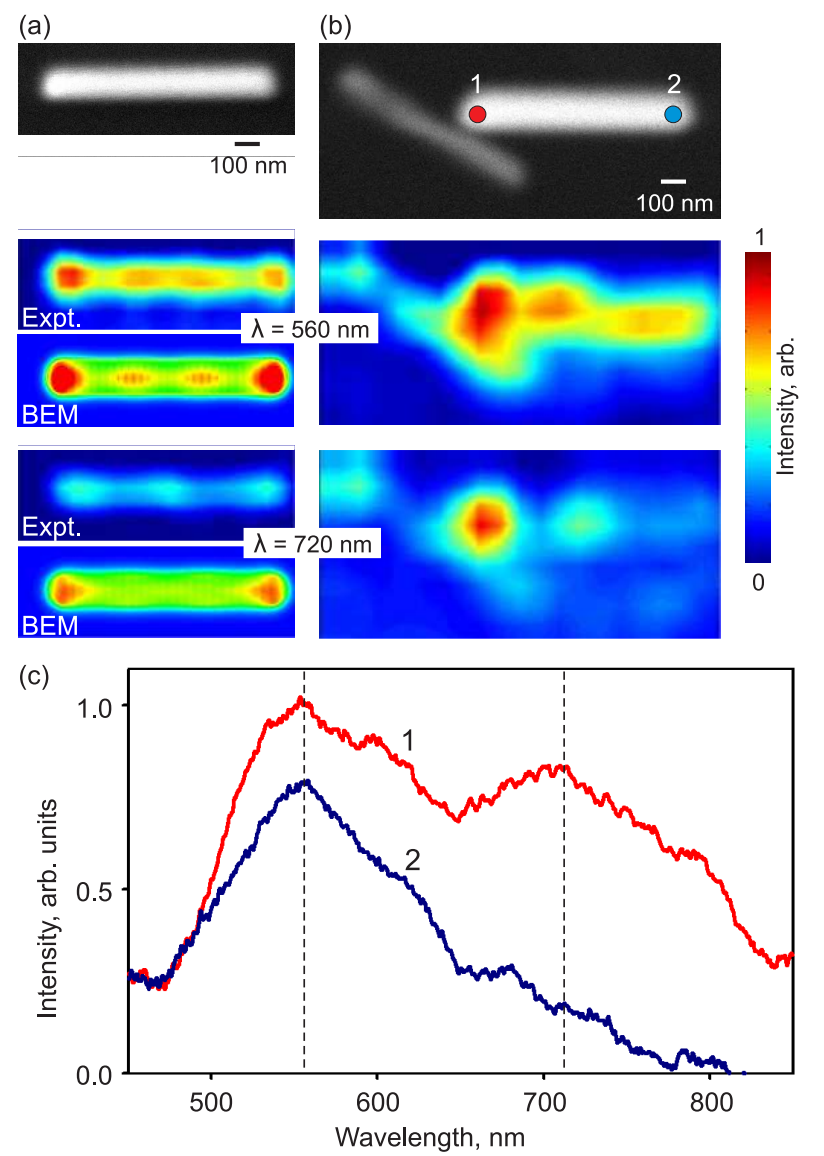

Figure 2: Scanning electron microscope (secondary electron) images and corresponding electron-beam-induced radiation emission (EIRE) excitation efficiency maps at 560 and $720 \mathrm{~nm}$ for (a) an isolated gold nanorod, alongside corresponding numerically simulated EIRE maps for a free-standing rod at these wavelengths; and (b) a pair of coupled gold nanorods including one rod with dimensions similar to those of the isolated rod in (a). (c) EIRE spectra for electron beam injection points 1 and 2 highlighted on the secondary electron image in (b). 
of plasmonic standing wave modes recently reported by Vesseur et al., ${ }^{11}$ with emission resonance wavelengths dictated by the dimensions of the rod. These emission characteristics are elegantly reproduced in numerical simulations employing a fully three-dimensional implementation of the boundary element method (BEM-3D) for rigorously solving Maxwell's equations. ${ }^{19,20}$ In coupled nanorod configurations light emission is found to be substantially stronger for electron beam injection points in the vicinity of the rods' junction than for any other point on the structure (Figs. 2b and 3a,b). Consider for example the coupled nanorod pair shown in Fig. 2b, where the horizontally aligned rod has dimensions very similar to those of the isolated rod in Fig. 2a. The EIRE spectrum for the junction region shows two maxima at 560 and $720 \mathrm{~nm}$ (Fig. 2c). EIRE intensity maps at these wavelengths illustrate that light emission at $720 \mathrm{~nm}$ is particularly strongly enhanced: The emission intensity is at least two times higher for electron beam injection points in this region than for any other point on the nanorods, or indeed any point on the corresponding isolated rod.

The behavior of coupled metallic nanorods as optical antennae can be related to the spatial distribution of the electromagnetic 'local density of states' (LDOS) ${ }^{21}$ around the nanostructure. Indeed, while a direct reciprocal analogy cannot be drawn between the transmitting antennae presently under consideration and more familiar optically illuminated receiving antennae, the local field enhancements achieved at the junctions of the latter are a reflection the elevated LDOS in that region. ${ }^{4,22}$ It has recently been demonstrated that the LDOS of a sample can be probed with an electron beam via electron energy loss and cathodoluminescence spectroscopies. ${ }^{23,24}$ In the present case of coupled nanorod pairs, enhanced light emission is observed for electron beam injection points in the proximity of the junction between the rods, i.e. the region of increased LDOS (such as point 1 in Fig. 2b). This increased LDOS gives rise to enhanced electron energy loss leading to increased surface plasmon generation and ultimately light emission. In contrast, when the electron beam targets the distal end of a coupled rod (e.g. point 2 in Fig. 2b), the LDOS it encounters is lower (es- sentially the same as that which it would find in an isolated rod), and light emission is correspondingly weaker.

In the terminology of electrical engineering, the junction between the rods provides an optimal feed point analogous to the ideal feed location at the center of the dipole for a microwave antenna. Here, one should consider the beam of electrons not as a continuous stream of current in free space, but rather as individual electrons electrons flying across the junction one after another. Through the Coulomb interaction, which disturbs the equilibrium of the electron distribution, the passing electrons induce oscillating currents (plasmons) in the poles of the Hertzian antenna. The free-electron feed is coupled most efficiently to the antenna when the current oscillations are resonant with the radiating modes of the antenna, in this case in the optical part of the spectrum.

Figs. $3 \mathrm{a}$ and $3 \mathrm{~b}$ show respectively an SEM im-
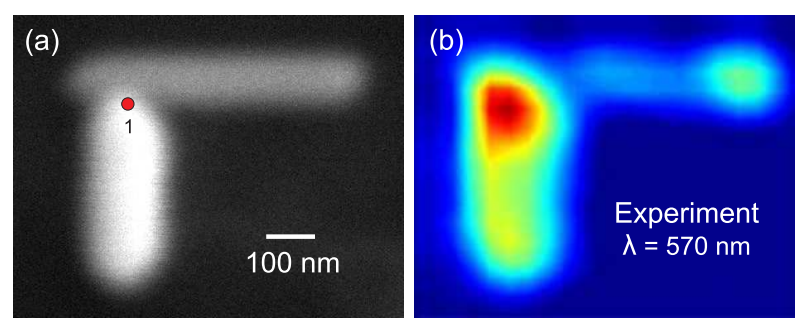

Intensity, arb.
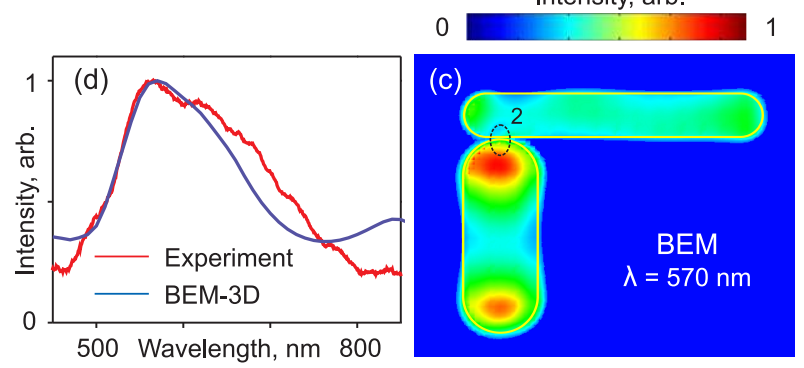

Figure 3: (a) Scanning electron microscope image and (b) corresponding EIRE excitation efficiency map at $570 \mathrm{~nm}$ for an $L$-shaped gold nanorod antenna; (c) Numerically simulated (BEM-3D) $570 \mathrm{~nm}$ EIRE map for such a configuration; (c) Normalized spectral dispersion of experimentally measured EIRE [red line] for an electron injection point at the junction between the rods [point 1 in part (a)] and numerically modelled EIRE [blue line] for the junction region [averaged over injection points at the centre of the junction and $10 \mathrm{~nm}$ either side - zone 2 in part (d) - to account for the real beam size in experiment]. 
age and an experimentally measured $570 \mathrm{~nm}$ EIRE map for a pair gold nanorods in a different geometric configuration, illustrating once more the increased strength of emission for injection points in the vicinity of the rods' junction. (The EIRE spectrum for electron injection at the junction is shown in Fig. 3d) It is apparent from the experimental examples presented in Figs. 2 and 3, and confirmed in BEM simulations of various antenna geometries similar to the experimental configurations (such as shown in Fig. 3c), that EIRE is maximized in these asymmetric antennae for electron injection points offset slightly from the centre point of the junction. This is to be expected in situations where junctions are formed between arbitrarily oriented rods of differing length and diameter, and typically between the end of one rod and the side-wall of the other: Under such circumstances the plasmonic modes of the two rods will have differing interactions with the junction and the LDOS is unlikely to be distributed symmetrically about the junction.

In summary, experiments have demonstrated that a pair of arbitrarily oriented coupled gold nanorods can act as a centre-fed resonant transmitting optical antenna, converting energy from a focused electron beam excitation into far-field light. Such free-electron pumped transmitting antennae are most efficiently feed in the vicinity of the junction between rods where the local density of electromagnetic states is elevated.

Acknowledgement The authors from the University of Southampton acknowledge the support of the UK Engineering and Physical Sciences Research Council (EPSRC, project EP/C511786/1), the European Union (FP6 project NMP4-2006016881, 'SPANS') and the Royal Society; Those from the University of Technology, Sydney thank the Australian Research Council and their institution; Those from the Instituto de Óptica - CSIC, Madrid acknowledge the European Union (NMP42006-016881) and the Spanish MEC (MAT200766050 and Consolider 'NanoLight.es').

\section{References}

(1) Greffet, J.-J. Science 2005, 308, 1561-1563.
(2) Brongersma, M. L. Nat. Photon. 2008, 2, 270.

(3) Taminiau, T. H.; Stefani, F. D.; Segerink, F. B.; Van Hulst, N. F. Nat. Photon. 2008, 2, 234-237.

(4) Aizpurua, J.; Bryant, G. W.; Richter, L. J.; García de Abajo, F. J.; Kelley, B. K.; Mallouk, T. Phys. Rev. B 2005, 71, 235420.

(5) Muskens, O. L.; Giannini, V.; SánchezGil, J. A.; Gómez Rivas, J. Opt. Exp. 2007, 15, 17736-17746.

(6) Yu, N.; Cubukcu, E.; Diehl, L.; Belkin, M. A.; Crozier, K. B.; Capasso, F.; Bour, D.; Corzine, S.; Hòfler, G. Appl. Phys. Lett. 2007, 91, 173113.

(7) Adamo, G.; MacDonald, K. F.; Fu, Y. H.; Wang, C.-M.; Tsai, D. P.; García de Abajo, F. J.; Zheludev, N. I. Phys. Rev. Lett. 2009, 103, 113901.

(8) Bashevoy, M. V.; Jonsson, F.; MacDonald, K. F.; Chen, Y.; Zheludev, N. I. Opt. Exp. 2007, 15, 11313-11320.

(9) Bosman, M.; Keast, V. J.; Watanabe, M.; Maaroof, A. I.; Cortie, M. B. Nanotech. 2007, 18, 165505.

(10) Yamamoto, N.; Nakano, M.; Suzuki, T. Surf. Interf. Anal. 2006, 38, 1725-1730.

(11) Vesseur, E. J. R.; de Waele, R.; Kuttge, M.; Polman, A. Nano Lett. 2007, 7, 2843-2846.

(12) Gómez-Medina, R.; Yamamoto, N.; Nakano, M.; García de Abajo, F. J. New J. Phys. 2008, 10, 105009.

(13) N'gom, M.; Li, S.; Schatz, G.; Erni, R.; Agarwal, A.; Kotov, N.; Norris, T. B. Physical Review B 2009, 80, 113411.

(14) Chu, M.-W.; Myroshnychenko, V.; Chen, C. H.; Deng, J.-P.; Mou, C.-Y.; García de Abajo, F. J. Nano Lett. 2009, 9, 399. 
(15) Bashevoy, M. V.; Jonsson, F.; Krasavin, A. V.; Zheludev, N. I.; Chen, Y.; Stockman, M. I. Nano Lett. 2006, 6, 1113-1115.

(16) van Wijngaarden, J. T.; Verhagen, E.; Polman, A.; Ross, C. E.; Lezec, H. J.; Atwater, H. A. Appl. Phys. Lett. 2006, 88, 221111.

(17) Nikoobakht, B.; El-Sayed, M. A. Chem. Mater. 2003, 15, 1957.

(18) Sau, T. K.; Murphy, C. J. Langmuir 2004, 20, 6414.

(19) García de Abajo, F. J. Rev. Mod. Phys. 2010, 82, 209-275.

(20) Myroshnychenko, V.; Carbó-Argibay, E.; Pastoriza-Santos, I.; Pérez-Juste, J.; LizMarzán, L. M.; García de Abajo, F. J. $A d v$. Mater. 2008, 20, 4288-4293.

(21) Dereux, A.; Girard, C.; Weeber, J.-C. J. Chem. Phys. 2000, 112, 7775-7789.

(22) Smolyaninov, I. I.; Zayats, A. V.; Gungor, A.; Davis, C. C. Phys. Rev. Lett. 2002, 88, 187402.

(23) García de Abajo, F. J.; Kociak, M. Phys. Rev. Lett. 2008, 100, 106804.

(24) Kuttge, M.; Vesseur, E. J. R.; Koenderink, A. F.; Lezec, H. J.; Atwater, H. A.; García de Abajo, F. J.; Polman, A. Phys. Rev. B 2009, 79, 113405. 
Graphical TOC Entry

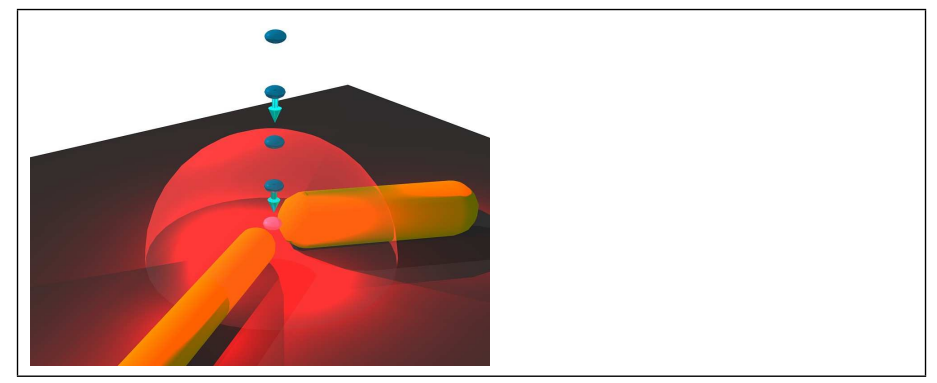

